\title{
Temperature Dependence of theTransport Properties in $\mathrm{ZnS}$
}

\author{
F.M.Abou El-Ela \\ Department of Physics, Faculty of Girls, Ain Shams Univ., Cairo, \\ Egypt
}

Transport properties of $\mathrm{ZnS}$ in an electric field have been calculated in the lattice temperature range between $77 \mathrm{~K}-500 \mathrm{~K}$, by using a Monte Carlo technique. It has been found that the threshold to occur a negative differential resistance changes over this temperature range. The threshold field increased from $1.5 \times 10^{5} \mathrm{~V} / \mathrm{cm}$ at $77 \mathrm{~K}$ to $2.4 \times 10^{5} \mathrm{~V} / \mathrm{cm}$ at $500 \mathrm{~K}$. The influence of impurity scattering event on $\mathrm{ZnS}$ has been neglected. 


\section{Introduction}

The transport properties of $\mathrm{ZnS}$ have been a subject of extensive investigation in recent years [1-7]. There has been a great interest in the study of charge carrier transport of $\mathrm{ZnS}$ due to the wide-band gap, which has become one of the most applied insulator in thin-film electroluminescence devices. Most previous studies have assumed the electron distribution function of cool temperature with high-energy tail ends at less than $4 \mathrm{eV}$. Muller et al. [3] have used a single parabolic band in the first conduction band. This is at very high field unrealistic. The full band details of the first two conduction bands as well as full-order treatment of the electron phonon interaction have been included by Brennan [2]. Electron Transport at low electric field was calculated for the first time by solving the Boltzmann transport equation, exactly under generalized Fermi-Dirac statistics for most scattering events. Electron mobility and resistivily were calculated as a function of electron concentration at both $300 \mathrm{~K}$ and $77 \mathrm{~K}$ [4]. Bhattacharyya et al. [5] used a model that included only the first conduction in a nonparabolic three valleys model. Fogarty et al. [6] have developed a model including a nonparabolic three valleys of the first conduction band and a single valley in the second conduction band. Using the pseudopotential method, the density of states in the last case was included. Most of the pervious theoretical work on transport properties of $\mathrm{ZnS}$, in high electric field, and at room temperature has been mainly based on Monte Carlo method.

The temperature dependence of the high field transport of $\mathrm{ZnS}$ is of particular relevance to the large effort at present to producing A.C and D.C electroluminscent devices. A.C Electroluminscent devices were made by encapsulating large band-gap semiconductors such as $\mathrm{ZnS}: \mathrm{Mn}$ or $\mathrm{ZnSe}: \mathrm{Mn}$ by two insulating layers, typically $\mathrm{Y}_{2} \mathrm{O}_{3}$, on either side of the semiconductor layers. An A.C bias applied across the device, alternately accelerates the electrons from one semiconductor insulator interface to the other. An electroluminescent phenomenon occurs when the free carriers are accelerated to sufficiently high energy, such that impact excitation of the Mn center becomes possible. For these reasons, we have calculated the temperature dependence of the transport properties using the ensemble Monte Carlo technique as has been previously used to determine the room temperature property [5-8]. Our calculations have been carried out for lattice temperature in the range of 77 to $500 \mathrm{~K}$.

\section{Monte Carlo Model}

The present computations of electron transport are based on Monte Carlo method. 4000 electrons have been used to simulate the transport 
properties through $\mathrm{ZnS}$ at steady state situation, in the range of lattice temperature of $77-500 \mathrm{~K}$.

A Monte Carlo simulation in semiconductors requires the material parameters, a knowledge of the energy band structure, the lattice temperature and the definition of the applied electric field. The process of simulating the carrier motion depends on a number of numerical computational steps to calculate the duration of the free flight, the type of scattering events and the choice of the state after scattering.

The main types of scattering processes included in our calculations are: polar optical phonon scattering, acoustic phonon scattering, equivalent intervalley phonon scattering, and non-equivalent intervalley phonon scattering

The state of an electron is specified by its wave vector $k$, which is related to the momentum $P$, and the electron energy $\varepsilon$ in non-parabolic conduction bands by

$$
\begin{gathered}
p=\hbar k \\
(1+\alpha \varepsilon)=\frac{\hbar^{2} \mathrm{k}^{2}}{2 \mathrm{~m}^{*}}
\end{gathered}
$$

where $\alpha$ is the band non-parabolicity given by,

$$
\alpha=\frac{1}{E_{g}}\left(1+\frac{m^{*}}{m_{0}}\right)
$$

where Eg is the energy gap between the conduction band and the valence band, $m^{*}$ is the electron effective mass in the material, $\hbar$ is plank's constant divided by $2 \pi$ and $\mathrm{m}_{\mathrm{o}}$ is the free electron mass.

The motion of the electron in an electric field $E$ is given by

$$
k(t)=k_{0}+\frac{q E \tau}{\hbar}
$$

where $q$ is the electron charge, $\tau$ is the free flight time, and $k_{o}$ is defined as the wave vector at time $t=0$.

When the electric field is applied in the $\mathrm{x}$ direction, the average drift velocity and the average electron energy are given for each valley, respectively, by,

$$
\overline{\mathrm{v}} \mathrm{d}=\frac{1}{\mathrm{~N}} \sum_{\mathrm{i}=1}^{\mathrm{N}} \mathrm{v}_{\mathrm{i}}(\mathrm{t}) \quad \bar{\varepsilon}=\frac{1}{\mathrm{~N}} \sum_{\mathrm{i}=1}^{\mathrm{N}} \varepsilon_{\mathrm{i}}(\mathrm{t})
$$


where

$$
\begin{aligned}
& \mathrm{v}_{\mathrm{i}}(\mathrm{t})=\frac{\hbar \mathrm{k}_{\mathrm{xi}}}{\mathrm{m}^{*}\left(1+2 \alpha \varepsilon_{\mathrm{i}}\right)} \quad, \quad \varepsilon_{\mathrm{i}}(t)=\frac{-1+\sqrt{1+4 \alpha \gamma(k)}}{2 \alpha} \\
& \text { and } \quad \gamma(k)=\frac{\hbar^{2}}{2 \mathrm{~m}^{*}}\left(\mathrm{k}_{\mathrm{xi}}^{2}+\mathrm{k}_{\mathrm{yi}}^{2}+\mathrm{k}_{\mathrm{zi}}^{2}\right)
\end{aligned}
$$

The sum in eq. (5) is over all electron numbers $(N)$ in each valley. $v_{i}(t)$ and $\varepsilon_{i}(t)$ represent the electron drift velocity and the electron energy at the end of each time step, while $\mathrm{k}_{\mathrm{xi}}, \mathrm{k}_{\mathrm{yi}}$ and $\mathrm{k}_{\mathrm{zi}}$ are the wave vector components in $\mathrm{x}$, $\mathrm{y}$ and $\mathrm{z}$ direction for each electron, respectively.

The model of band structure, the types of scattering mechanisms, and the parameters used in our calculation are the same as described previously $[5,7]$. The Monte Carlo procedure used in this work is based on the constantfield technique of references [8-11] with non-parabolic three-valleys $\Gamma, \mathrm{L}, \mathrm{X}$ model.

The influences of ionized impurity scattering, neutral impurity scattering, impact ionization scattering, and piezoelectric scattering have been ignored since these scattering processes are very weak compared to other scattering processes at this early stage of our attempt to model the transport properties of $\mathrm{ZnS}[5,6]$

We have neglected screening of the polar mode interaction; in fact, this is due to the low carrier concentration in $\mathrm{ZnS}$ samples. Also, in our calculations, the admixture of p-type wave function of the valence bands into the wave function of the conduction bands has been ignored at electron energies greater than $1 \mathrm{eV}[8]$.

\section{Results and discussion}

The total average drift velocity has been calculated by

$$
\mathrm{v}_{\mathrm{t}}=\frac{\mathrm{n}_{\Gamma} \mathrm{v}_{\Gamma}+\mathrm{n}_{\mathrm{L}} \mathrm{v}_{\mathrm{L}}+\mathrm{n}_{\mathrm{X}} \mathrm{v}_{\mathrm{X}}}{\mathrm{N}_{\mathrm{t}}}
$$

where $\mathrm{V}_{\Gamma}, \mathrm{V}_{\mathrm{L}}$ and $\mathrm{V}_{\mathrm{X}}$ are the electron average drift velocity in $\Gamma, \mathrm{L}$, and $\mathrm{X}$ valleys, respectively. $n_{\Gamma} n_{L}$, and $n_{X}$ are numbers of the electrons in $\Gamma$, L, and $X$ valleys, respectively. $N_{t}$ is the number of electrons in all valleys. 
Figure (1) shows the calculated average drift velocity for electrons as a function of electric field strength for various lattice temperatures. It is seen that the peak velocity and the low field mobility rise as a result of temperature cooling; in fact, this is due to the low phonon occupation number,

$$
N_{p o}=\left[\exp \left(\frac{\hbar \omega_{p o}}{k_{B} T}\right)-1\right]^{-1}
$$

$\left(\omega \hbar_{\mathrm{po}}\right.$ refers to the optical phonon energy, $T$ represents lattice temperature and $k_{\mathrm{B}}$ defines as a Boltzmann's constant); additionally, the small phonon occupation number lowers phonon scattering rates. The absence of phonon absorption at $77 \mathrm{~K}$ also explains the extremely high low field mobility. The probability of polar optical phonon emission for a given event is zero unless the electron energy at least equals the energy of one phonon. A polar optic phonon energy in $\mathrm{ZnS}$ is $0.044 \mathrm{eV}$, while thermal average energy $\left(k_{\mathrm{B}} \mathrm{T}\right)$ is $0.0067 \mathrm{eV}$ for $77 \mathrm{~K}$. Since, the scattering rate at low electron energy is so small, the electron distribution can be expected to be thermalised

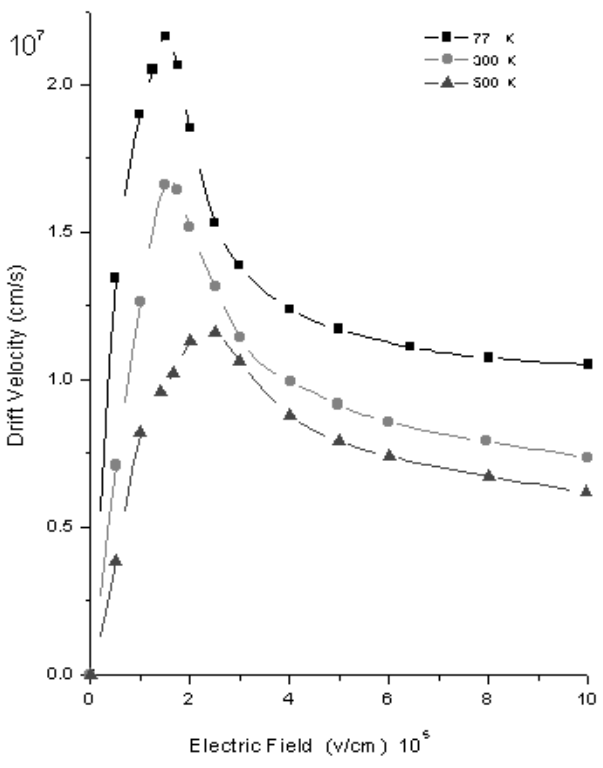

Fig.(1) Drift electron vel ocity versus electric field for various lattice temperatures

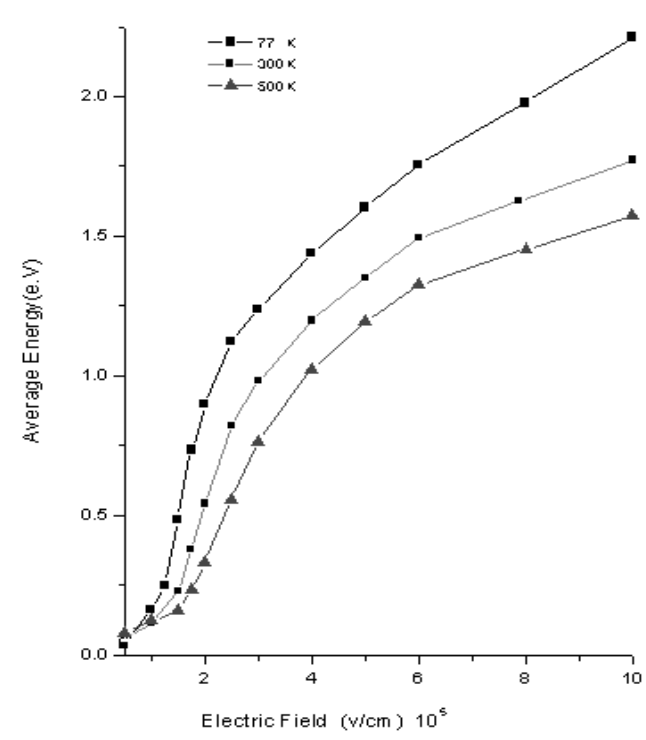

Fig.(2) Average electron energy versus electric field for various lattice temperatures 
high low-field mobility can be seen at lattice temperature $77 \mathrm{~K}$.

The term $\left(N_{p o}+1\right)$ represents spontaneous and stimulated phonons emission, while $N_{p o}$ takes into account phonon absorption.

Also it can be seen that a slight dependence of lattice temperatures on the threshold field occurs for onset of negative differential mobility. The threshold field varies from $1.5 \times 10^{5} \mathrm{~V} / \mathrm{cm}$ at $77 \mathrm{~K}$ to $2.4 \times 10^{5} \mathrm{~V} / \mathrm{cm}$ at $500 \mathrm{~K}$.

The total average electron energy has been calculated by

$$
\bar{\varepsilon}_{\mathrm{t}}=\frac{\mathrm{n}_{\Gamma} \bar{\varepsilon}+\mathrm{n}_{\mathrm{L}} \bar{\varepsilon}_{\mathrm{L}}+\mathrm{n}_{\mathrm{X}} \bar{\varepsilon}_{\mathrm{X}}}{\mathrm{N}_{\mathrm{t}}}
$$

where $\bar{\varepsilon}_{\Gamma}, \bar{\varepsilon}_{\mathrm{L}}$ and $\bar{\varepsilon}_{\mathrm{X}}$ are the electron average energy in $\Gamma$, L, and $\mathrm{X}$ valleys, respectively.

The total average electron energy is represented in Fig. (2). The average energy at $77 \mathrm{~K}$ is small compared to those at $300 \mathrm{~K}$ and $500 \mathrm{~K}$. This is due to small phonon absorption rates at low lattice temperatures. As a consequence, the fractional number of electrons in $\Gamma$ valley $\left(\mathrm{n}_{\Gamma} / \mathrm{N}_{\mathrm{t}}\right)$ at low lattice temperatures is relatively high compared to that at high lattice temperatures. This can be seen in Fig. (3).

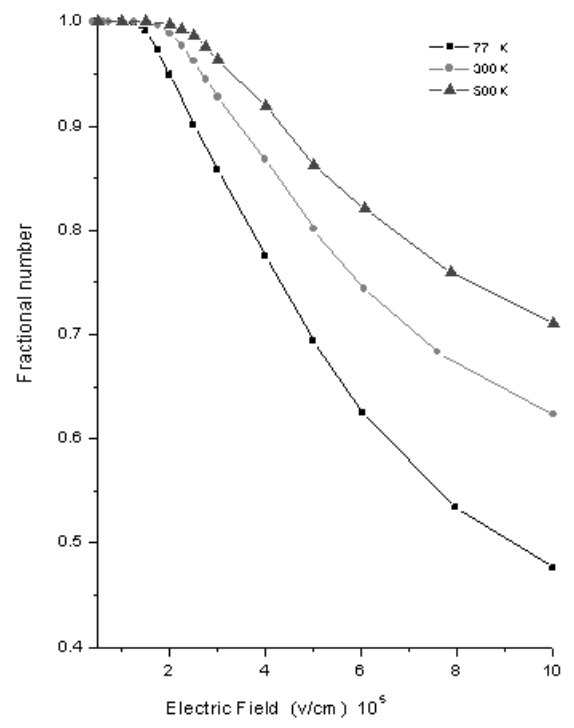

Fig.(3) Electron fractional number versus electric fielf for various lattice temperatures

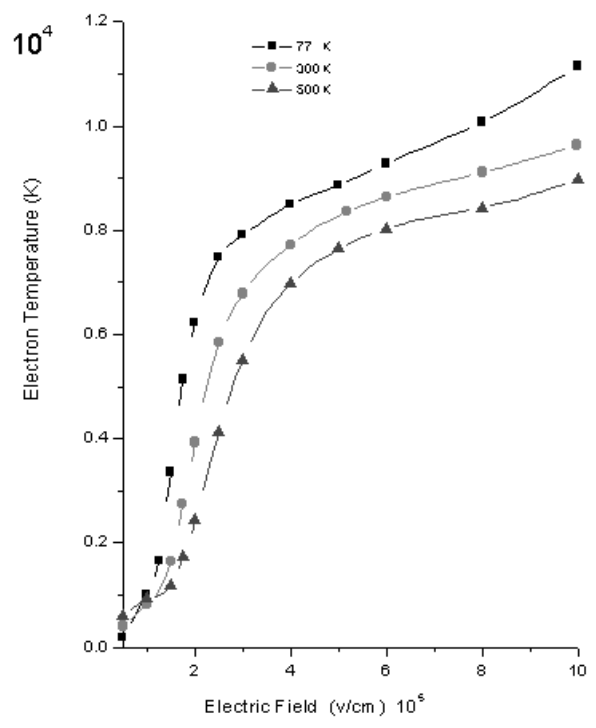

Fig.(4) Average electron temperature versus electric field for various lattice temperatures

The variation of electron temperature as a function of electric field strength at various lattice temperatures is presented in Fig. (4). The electron 
temperature, $T_{e}$, was collected during the Monte Carlo computer run for steady state by setting the average carriers energy $\left(\bar{\varepsilon}_{\mathrm{t}}\right)$ minus the carrier drift energy $\left(1 / 2 \mathrm{~m} * \mathrm{v}_{\mathrm{t}}^{2}\right)$ equals to $2 / 3 \mathrm{k}_{\mathrm{B}} \mathrm{T}_{\mathrm{e}}[10]$.

The rapid increase of electron temperature above the threshold electric field is a consequence of decreasing effectiveness of the polar optical phonon mechanism at electron energies above $1 \mathrm{eV}$.

Although, the electric field becomes high, the number of electrons in $\Gamma$ valley is still low. However, the electron temperature is extremely high, and the electrons are communicated to both $\mathrm{L}$ and $\mathrm{X}$ valley electrons after the intervalley scattering becomes more effective; and thus, causing their high field temperature to increase. Otherwise, the $\mathrm{L}$ and $\mathrm{X}$ valley electron temperatures are substantially constants, because of the dominance of equivalent intervalley scattering and the absence of the breakdown of polar optical phonon scattering.

The different types of phonon scattering events have been analyzed in Figs. (5a-5d). Each type of scattering event has been recorded according to the type of each conduction band minimum. The number of each scattering mechanism was normalised by the number of all types of scattering events, which is defined as the fractional scattering number (F.N.S).

The variation of the average fractional scattering number (F.N.S) of acoustic phonon scattering, polar optical phonon scattering, and nonequivalent intervalley phonon scattering events against the electric fields are represented in Figs. (5a-5d). The fraction scattering number of acoustic scattering is very small at low lattice temperatures as shown in Fig. (5a). On the other hand, the fractional number of polar optical phonon emission scattering is larger at low lattice temperatures than that at high lattice temperatures. On contrary, the fractional number of polar optical absorbed phonon at high lattice temperatures is large than at low lattice temperatures as shown in Fig. (5b). Finally, the fractional scattering number of intervalley scattering in both emission and absorption are presented in Figs. (5c-5d). The lattice temperature varies in the range $(77 \mathrm{~K}-500 \mathrm{~K})$ in the case of $\Gamma-\mathrm{X}$ and $\Gamma-\mathrm{L}$ transition, respectively. It is obvious to notice the large fractional scattering number at low lattice temperatures compared to high lattice temperatures in the case of emission phonons, while the opposite occurs in the case of absorption phonons.

As a conclusion, the threshold field for negative differential resistance (NDR) to occur in $\mathrm{ZnS}$, is changed over the lattice temperature range $77 \mathrm{~K}$ $500 \mathrm{~K}$. The threshold field varies from $1.5 \times 10^{5} \mathrm{~V} / \mathrm{cm}$ at $77 \mathrm{~K}$ to $2.4 \times 10^{5} \mathrm{~V} / \mathrm{cm}$ at $500 \mathrm{~K}$. The absence of phonon absorption at $77 \mathrm{~K}$ explains the extremely high low-field mobility at that temperature. Also, the average electron energy 
increases with the lattice temperature cooling as a consequence of the lower phonon emission scattering rate.

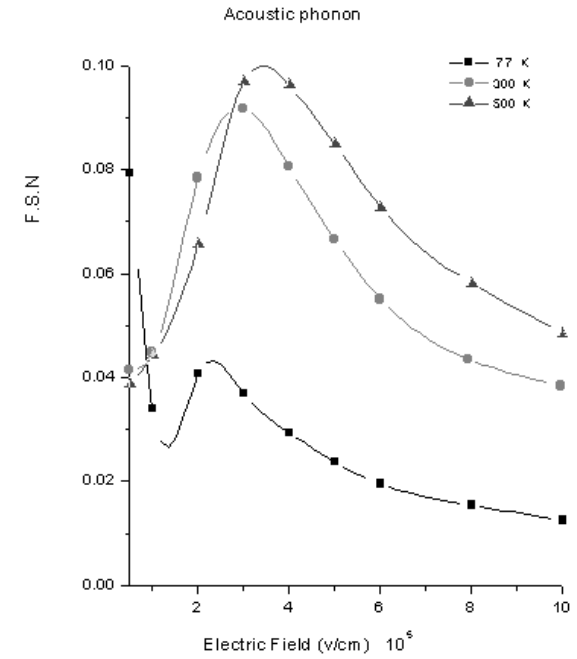

(a)

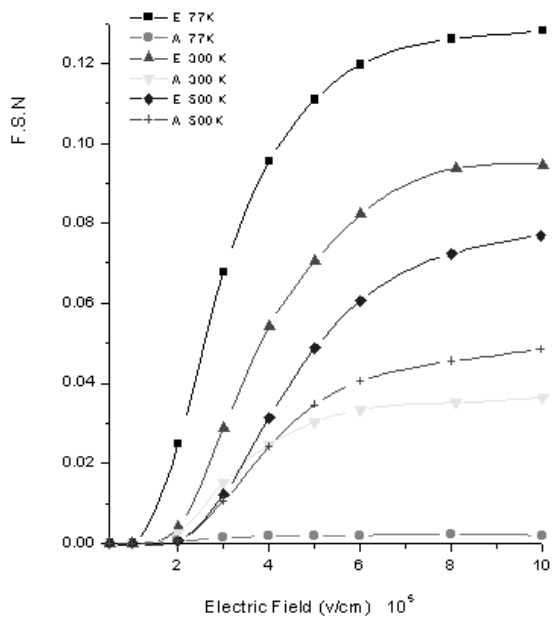

(c)

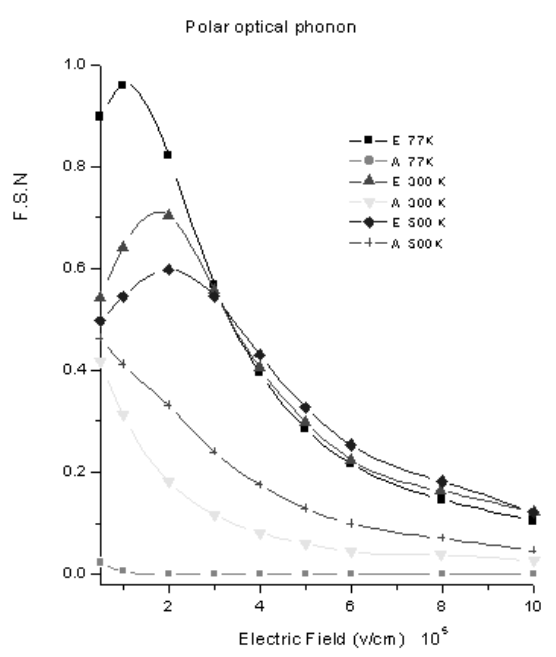

(b)

Fig. (5) : The average fractional scattering number for various scattering mechanisms.

\section{Acknowledgments}

The author would like to thank Prof. H.Talaat at Ain Shams University, Faculty of science, Department of Physics for helpful discussion and for critical reading of the manuscript. 


\section{References}

1. B.K Ridley and F.M.Abou El-Ela, Solid State Physics conference, University of Bristol, Bristol, England, G9 (1987).

2. K. Brennan, J. Appl. Phys. 64, 4024 (1988).

3. H.J. Fitting, G.O. Muller, R. Mach, G. U. Reinsperger, T. Hingst, and E. Schreiber, Phys. Status Solidi A 121,305 (1990).

4. H.E Ruda and B Lai, J. Appl. Phys. 68 (1990).

5. K. Bhattacharyya, S.M Goodnick and J.F Wager, J. Appl. Phys. 73, 3390 (1993).

6. J.Fogarty, W.Kong and R.Solanki, Solid State Electronics 38, 653 (1995).

7. F.M.Abou EL-Ela, to be published in Egyptian Journal of Solids

8. W. Fawcett, A.D.. Boardman and S. Swain, J. Phys. Chem. Solid 31,1963 (1970).

9. B .R. Nag "Electron Transport in Compound S.C.” Springer-Verlag Berlin, New York (1980).

10. T.J. Maloney and J. Frey, J.Appl. Phys. 48, 781 (1977).

11. J.G.Ruch and W. Fawcett, J.Appl. Phys. 41, 3843 (1970). 